\title{
An Equivalent of XOR Operator and Its Application in One- Dimensional CA Model
}

\author{
Youssef Khmou \\ Sultan Moulay Slimane University, Morocco. \\ Khmou.y@gmail.com
}

\begin{abstract}
In this paper, we study the probabilistic characterization of one dimensional cellular automaton model based on two values $\{0,1\}$. We propose a complex metric in order to characterize a one dimensional lattice with respect to the occupation probability also known as density. Based on numerical results, the proposed function is equivalent to XOR operator for two dimensional vector and for large system, it characterizes two phases with critical probability of $p=0.5$ which is the same result obtained using the information entropy function. In the second part, we study the variation of the proposed metric and the information entropy with respect to the repartition of the binary sequence that is generated using an optimal transformation of the logarithmic logistic map which is a non linear model of discrete dynamical system, numerical results illustrate the equivalence of the variations between both functions.
\end{abstract}

Keywords: Cellular automaton, binary, one dimensional, entropy, probability.

\section{Introduction}

Cellular automaton [1] consists of grid of cells with discrete states, it is used to model dynamical systems, and each cell can be in one of finite number of states which is a discrete ensemble. For example the binary configuration is defined by states 1 and 0 . The grid is implemented to model a system governed by rules expressed by mathematical functions, the dynamics of the cells are based on the rules relating the state of the cell $i$ at time index $t$ to the past state at time index $t-1$ and to the states of the neighbor cells for two dimensional grid, the states of all cells are updated simultaneously. The applications of cellular automata include several fields such as geography $[2,3]$ and computer science [4]. In vehicular traffic flow [5], the principle of cellular automata are based on the microscopic description of traffic by analyzing the displacement and velocity of each car, the macroscopic variables of the traffic such as global velocity, flow and density are derived based on the average of the microscopic variables. This principle allowed reproducing the observed phenomena in traffic flow.

In this paper, we study a particular property of one dimensional and binary cellular automaton model which consists of the characterization of the number of possible states with respect to the occupation probability; we propose a new function based on complex exponential to evaluate the probabilistic state of the one dimensional lattice, comparatively to the information entropy function. In the second part, we compare the response of the proposed metric and the information entropy function to the variation of the repartition of the binary sequences obtained using an optimal transformation of the logarithmic logistic map where the growth parameter is defined by the range [0,e]. As perspective, we present an application of the proposed metric $\Psi$ in particular case of deterministic traffic flow with periodic boundary conditions where each site of the lattice can either be empty or occupied by single vehicle. Based on the fundamental diagrams of

Received (October 3, 2017), Review Result (December 22, 2017), Accepted (January 4, 2018) 
flow-density and average velocity-density, the variation of $\Psi$ is proportional to the equation of the flow for particular value of the allowed maximum velocity.

\section{One Dimensional CA Model}

We consider a one dimensional cellular automaton model $x$ of length $L$, each cell can be occupied or empty with values 1 and 0 respectively, the occupation of cell represents different entities such as physical model as vehicles with same dimensions where $x$ is the section of road, or particle [6] with one dimensional movement in forward or backward direction on segment. The state of each cell is updated using predefined rules. In our case, we focus on the initial repartition of the particles and we analyze the state of the system based on information entropy function. Given a number of particles $N$ with the condition $0 \leq N \leq L$ such that the particles are randomly distributed on the grid, the probability of presence is equal to the density $\rho$ that is defined by the following relation:

$$
\rho=p(1)=\frac{N}{L}
$$

Consequently the probability of absence is defined by the following equation:

$$
p(0)=1-\rho=\frac{L-N}{L}
$$

For different values of the probability of presence starting from $p(1)=0$ to $p(1)=1$ with step of $d p=0.01$, we present in Figure.1, a random configurations showing the effect of linear increase of density for one dimensional system of length $L=200$.

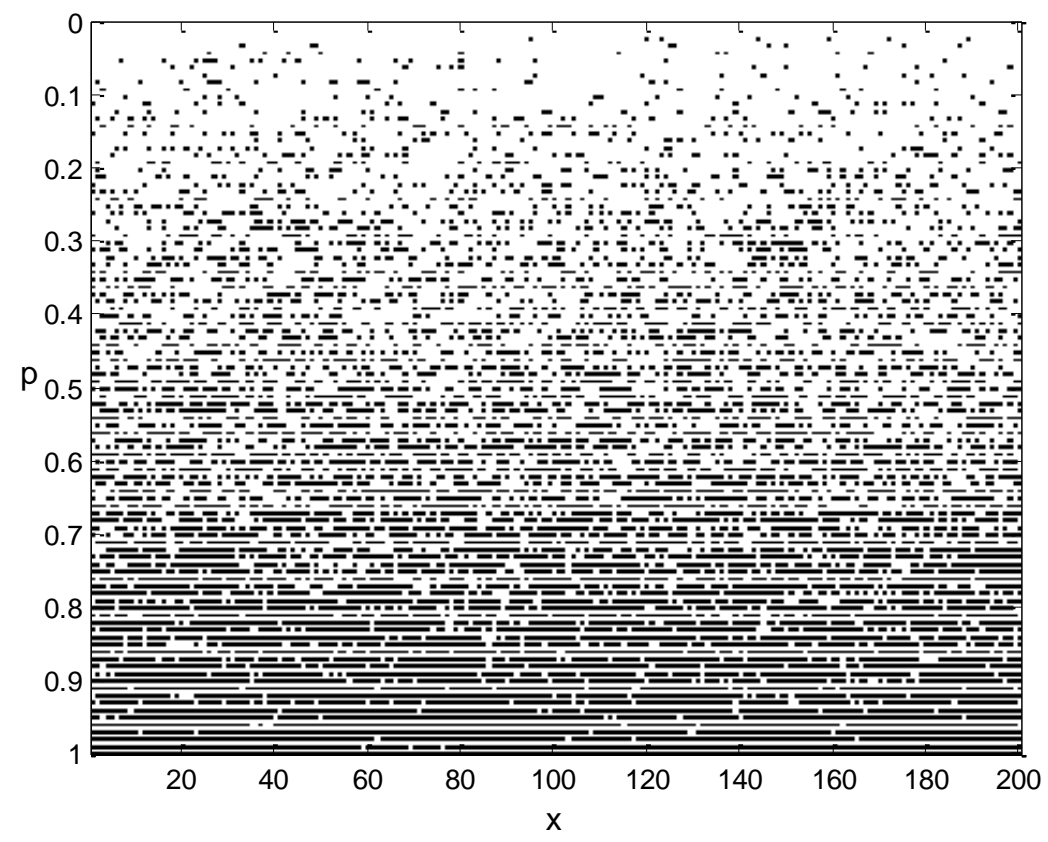

Figure 1. Different States, Randomly Distributed, of One Dimensional Cellular Automaton Model with Respect To the Probability $p(1)$ 
Note that for the case of $p(1)=0$, all the sites are empty which corresponds to the line at the top of the figure, and for $p(1)=1$ all the sites are occupied. By considering a value of the occupation probability, among the basic principles of the dynamics of the system is uniform displacement that consists of one directional movement of each particle such that the system represents a closed circuit which implies the condition of periodic boundaries, given a particle at position $n$ at time index $t$, its position at time step $t+1$ is advanced by one site $n+1$. Based on this rule we obtain a diagram of the positions and time steps represented by binary matrix $X \in N^{L \times T}$ where $L$ is the length of the chain and $T$ is the number of the time steps. To illustrate this principle, we present in figure.2, an example of system with $T=200$ time steps starting from index $t_{0}=200$ and with occupation probability of $\rho=0.05$.

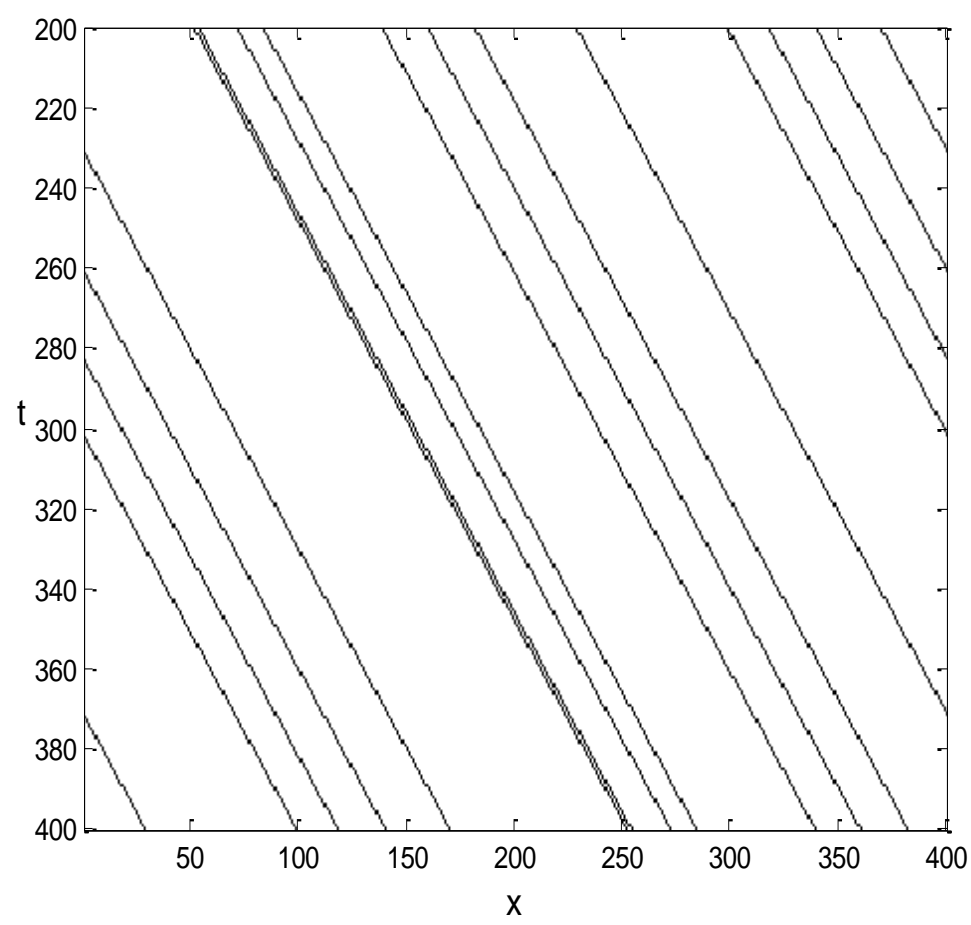

Figure 2. Elementary Example of Discrete 1D Dynamical System with Length $L=400$ and Constant Density $\rho=0.05$

A scalar function that can be used to evaluate the probabilistic state of the system $x$ is Shannon entropy, which is function of the probabilities $p(1)$ and $p(0)$, it is given by the following equation:

$$
h=-p(1) \log (p(1))-p(0) \log (p(0))
$$

The metric $h$ can also be expressed as function of density only, using the relation $\rho=1-p(0)$, we obtain the second expression as the following:

$$
h=\rho \log \left(\frac{1-\rho}{\rho}\right)-\log (1-\rho)
$$

To compute the probability of occupation, we simply sum the states of the system $x$ as the following: 


$$
p(1)=\frac{1}{L} \sum_{n=1}^{L} x_{n}
$$

We suppose that the system is dynamical such that the density changes with respect to some constraints, a critical point exists such that both the probabilities are equal, it corresponds to a state where the number of occupied cells equals the number of empty cells:

$$
p(1)=p(0)=\frac{1}{2}
$$

In terms of density, this critical point corresponds to the critical density which can be computed using the number of particles with $L+1$ distinct values:

$$
\rho_{c}=\frac{1}{L(L+1)} \sum_{N=0}^{L} N=\frac{1}{2}
$$

In this state, the entropy $h$ is maximal and equals $\max (h)=\log (2)$, this implies that the entropy of the system is maximal when the probability coefficients are equal, however the function $h$ cannot describe whether the state of the system is random or not, for this purpose, other functions are necessary to quantify the randomness. Our contribution is to propose an alternative function for verifying the state of the system from probabilistic viewpoint similarly to the information entropy. We consider a function $\Psi$ defined as follows:

$$
\Psi=1-\left|<e^{j \pi x}>\right|=1-\left|\frac{1}{L} \sum_{n=1}^{L} e^{j \pi x_{n}}\right|
$$

Where |. is the absolute values, the function $\Psi$ is based on the values of the complex exponential using the binary states of the cells as inputs, thus we have the two output values $e^{j \pi}=-1$ and $e^{0}=1$. To evaluate the function $\Psi$, we begin with elementary example of system with two cells $\mathrm{x}=\left[x_{1}, x_{2}\right]$, the number of the possibilities of this system is $2^{2}$, by examining the values of $\Psi$ with respect to the four states, we obtain the following results comparatively to the information entropy and exclusive $O R$ operator.

Table 1. Output Values of Information Entropy $h$, Metric $\Psi$ and XOR Operator for Two Dimensional Binary System $x$.

\begin{tabular}{|c|c|c|c|c|}
\hline$x_{1}$ & $x_{2}$ & $h$ & $\Psi$ & $X O R$ \\
\hline 0 & 0 & 0 & 0 & 0 \\
\hline 0 & 1 & $\log (2)$ & 1 & 1 \\
\hline 1 & 0 & $\log (2)$ & 1 & 1 \\
\hline 1 & 1 & 0 & 0 & 0 \\
\hline
\end{tabular}

The XOR operator is defined by $X O R\left(x_{1}, x_{2}\right)=x_{1} \oplus x_{2}=\overline{x_{1}} x_{2}+x_{1} \overline{x_{2}}$. We remark that $\Psi$ for a system $x$ with two cells is equivalent to $X O R$ operator and the response of $\Psi$ is similar to $h$ from probabilistic view point since the order of theirs output values follows the same pattern with respect to the probability coefficients as explained by the following table. 
Table 2. Probability Coefficients of the Binary Values of System with Two Cells

\begin{tabular}{|c|c|}
\hline$p(0)$ & $p(1)$ \\
\hline 1 & 0 \\
\hline $1 / 2$ & $1 / 2$ \\
\hline $1 / 2$ & $1 / 2$ \\
\hline 0 & 1 \\
\hline
\end{tabular}

For quantitative comparison, we consider a large system that consists of $L=400$ cells, each cell is occupied with probability $p(1)$, by varying the probability of occupation with step $d p=0.01$, we compute the information entropy $h$ and our function $\Psi$ where we takes theirs averages over $\mathcal{M}=00$ trials for each value of $p(1)$, the simulation is based on the convention $0 \log (0)=0$ for the information entropy $h$ and the values of the system $x$ are generated using uniform random number variable $x_{i}$ such that if $x_{i}<p(1)$ then $x_{i}=1$ else $x_{i}=0$, the results are presented in Figure. 2 where the function of the entropy is scaled to have a maximum value of unity using the operation $h / \log (2)$.

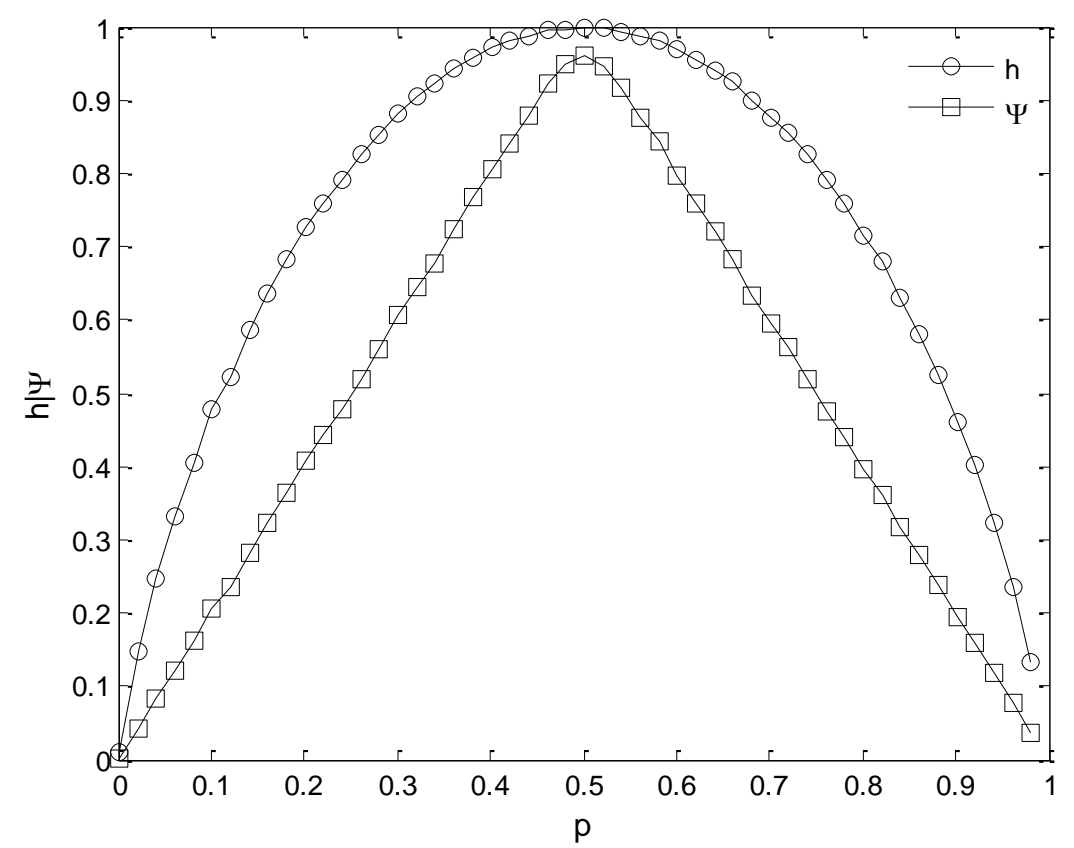

Figure 3. Variations of the Function $\Psi$ and Information Entropy $h$ with Respect to the Occupation Probability $p(1)$ for One Dimensional System of Length $L=400$

From the result, we remark that both function characterize two phases of the variation of the system, in the range $p \in[0,1 / 2]$ the number of the possibilities of the system $x$ increases where the rate is linear for the metric $\Psi$ and non linear for the information entropy $h$. In the range $p \in[1 / 2,1]$, the number of the possible states of the system decreases with linear and non linear rates for $\Psi$ and $h$ respectively, both functions have single peak where $\max (\Psi)=1$ and 
$\max (h)=\log (2)$ (note that in the figure, the maximum value of $h$ is normalized). The result is based on the average of $M$ trials, we can test how the functions fluctuate around theirs average values, for this purpose we choose two values of the occupation probability $p(1)=0.1$ and $p(1)=0.5$, we plot in Figure.4, the outputs of $\Psi$ and $h$.

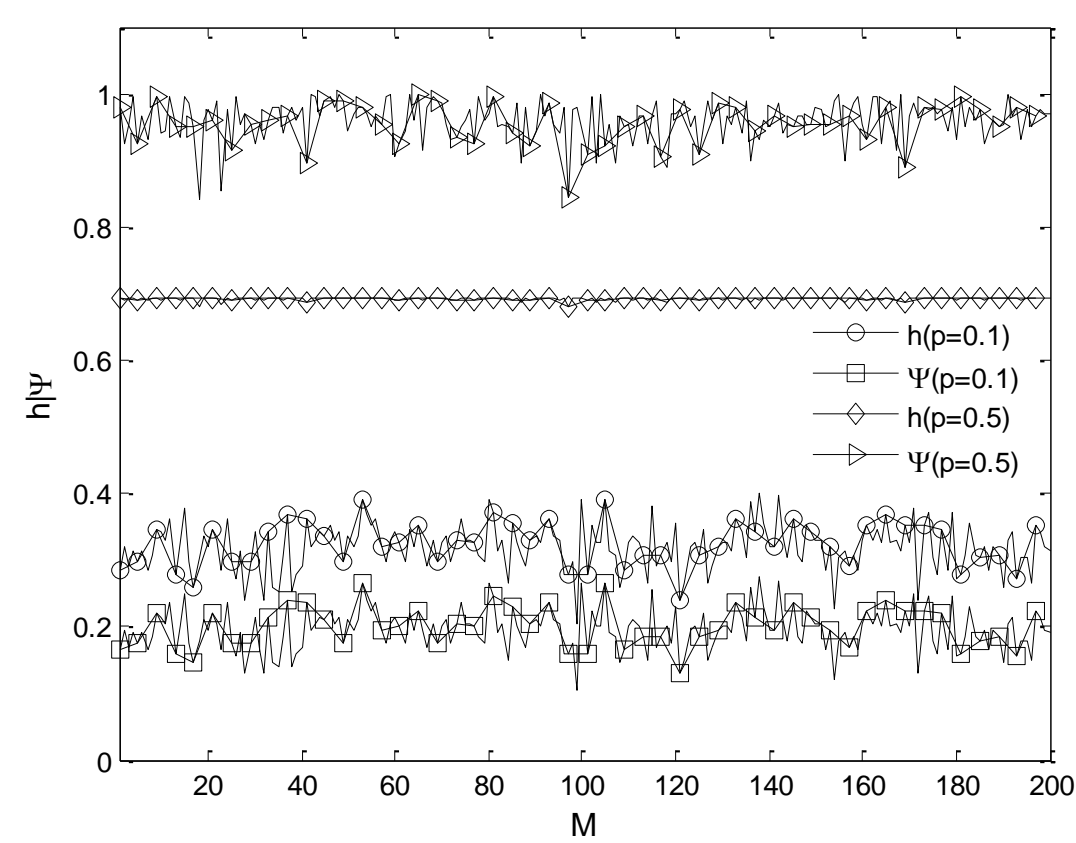

\section{Figure 4. Variations of the Functions $\Psi$ and $h$ with Respect to the Number of Trials $M$ for Occupation Probabilities $p(1)=0.1$ and $p(1)=0.5$}

We remark that for $p(1)=0.1$, the values of $\Psi$ and $h$ change such that theirs fluctuations are approximately the same, for the case of $p(1)=0.5$, the information entropy function is stable and $\Psi$ is characterized by dispersion less than 0.2 . From these simulation results, we conclude that the application of the function $\Psi$ for probabilistic characterization of the system $x$ is valid similarly to the information entropy. In the next part, we compare the response of the functions $\Psi$ and $h$ in the context of random binary sequences.

\section{Application in Random Binary Sequences}

The one dimensional cellular automaton model $x$ discussed in the previous section consists of $L$ sites where each site can either be occupied or empty. The repartition of the $N$ particles on $L$ sites can be generated using different methods. In this section, we consider the methodology that is based on logistic map which is a model of non linear discrete dynamical system.

To characterize the properties of such systems, bifurcation theory [7] allows investigating different phases of the evolution of the map functions. Indeed the bifurcation theory has been applied in several physics field, including for example laser dynamics [8] and quantum chaos [9]. The characterization of the map functions relies on the structure of the bifurcation diagram which shows the impact 
of the parameters of the model, which can be the transition from period doubling phase to chaotic behaviors [10].

The chaotic behavior implies that the model of the map function is sensitive to the initial conditions, such that starting with two values of the pair $\left(x_{0}, \mu\right)$ with infinitesimal differences makes the later values of the system for the two cases $x_{n}$ different [11]. The chaotic behavior is measured with Lyapunov coefficient [12] $\lambda$ which is also a function of the parameter $\mu$, positive value of $\lambda$ implies that the system is chaotic, negative value represents stable and periodic system, the case of $\lambda=0$ implies marginally stable orbits.

Several other metrics were proposed to investigate the chaotic behaviors of the logistic maps such as information entropy [13] where for each value of parameter $\mu$, the entropy function $h$ of the sequence $x_{n}$ is computed leading to spectrum $h(\mu)$ such that the bifurcations are represented by discontinuities. We consider the repartition of the particles using the logarithmic logistic map [14] which is defined by the following first order iterative equation:

$$
x_{n+1}=-\mu x_{n} \log \left(x_{n}\right)
$$

Defined with initial condition $x_{1} \in[0,1]$ where the range of the growth parameter is $\mu \in[0, e]$ and the range of the sequence is $0 \leq x_{n} \leq 1$. In Figure.5, we present the bifurcation diagram where different phases of the system are illustrated, the last 200 points from $M=4000$ iterations are considered to ensure that the system reaches stationary state and for better presentation of the diagram.

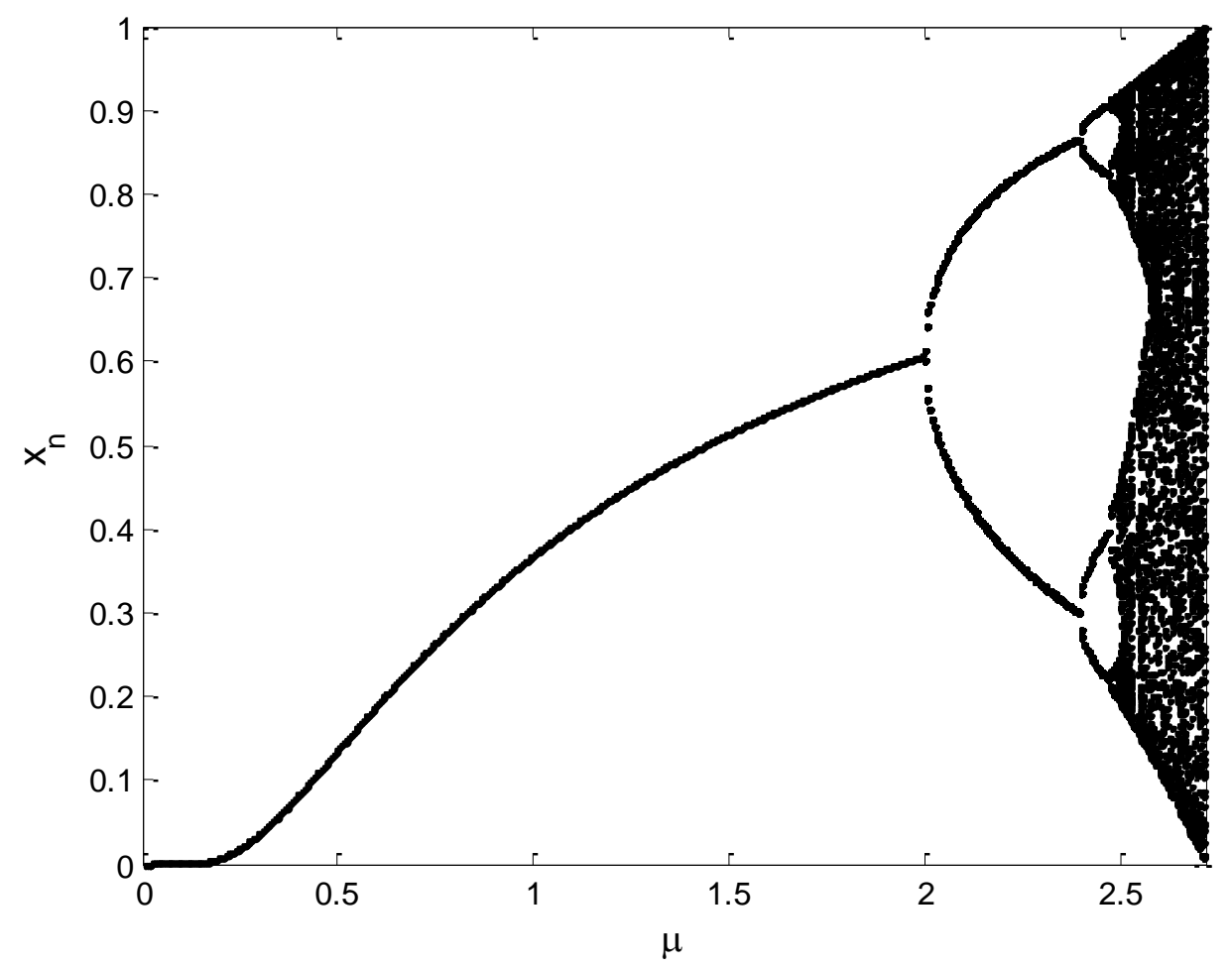

Figure 5. Bifurcation Diagram of the Logarithmic Logistic Map 
For given value of the growth parameter, we transform the sequence $x_{n}$ into binary sequence using the optimal value $d=0.5$ as the threshold, the transformation is given by the following rule:

$$
y_{n}=\left\{\begin{array}{lll}
1 & \text { if } & x_{n}>d \\
0 & \text { if } & x_{n}<d
\end{array}\right.
$$

Next, we consider a lattice of length $L=2000$, where the repartition of the $N$ particles is described by the rule given in equation (10), for each value of the parameter $\mu$, a lattice $x$ is generated using the sequence $y_{n}$ computed using the last 2000 points of $M=4000$ trials of $x_{n}$. For each value of $\mu$, the information entropy function $h$ and the metric $\Psi$ are computed, we obtain two characteristic functions that we present in the following figure.

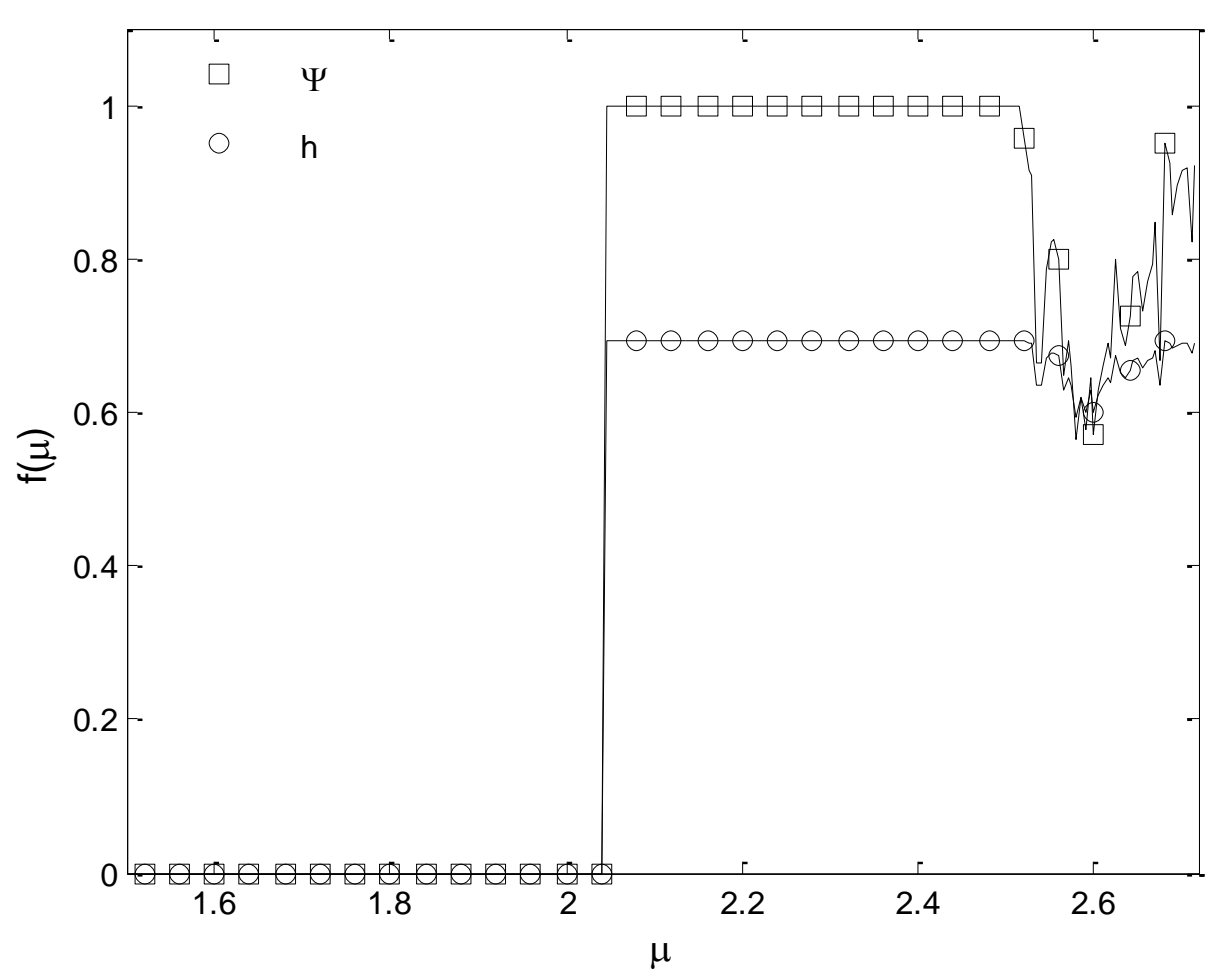

\section{Figure 6. Variations of the Metrics $\Psi$ and $h$ as Function of the Generated Binary Sequences using logarithmic Logistic Map, with Respect to the Growth Parameter $\mu$}

We remark that the values of the functions equal zero in the range $\mu \in[0,2]$ with sharp transition at approximately the index that corresponds to the first bifurcation point of the logarithmic logistic map given by $(2,0.6)$. At this point, the functions reach their maximum values 쟁 $\mathrm{g}(2)$ and 1 for $h$ and $\Psi$ respectively.

The chaotic region of this map is defined by the range $\mu \in[2.5, e]$, the responses of the functions $\Psi$ and $h$ are characterized by fluctuations. The amplitude of the fluctuation of the function $\Psi$ is higher than that of the entropy function, as the growth parameter tends 
to $e$, the functions tend to their maximum values. From this simulation result, we consider that the complex metric $\Psi$ is able to quantify the change of the binary sequence obtained from the logarithmic logistic map similarly to the Shannon entropy. In the next part, we present a particular application of the metric $\Psi$ in traffic flow analysis.

\section{Application in Deterministic Traffic Flow}

Vehicular traffic flow [15] can be studied using different variables such as average velocity, occupancy, kinetic energy, flow known also as current, space-time diagrams and short range correlation functions [16]. Each type of traffic flows is defined by its own characteristics such as critical density, open or periodic boundary conditions and the types of vehicles. The characteristics of the flow are based on macroscopic variables including average velocity $\langle v\rangle$, density $\rho$ and flow $J$, and different fundamental diagrams of the flow are obtained using the representations $(\rho, J),(\rho,<v>)$ and $(J,<v>)$ where for each diagram different phases of circulation can be defined. Based on deterministic model [15] of the traffic flow, the fundamental diagram of the flow-density is given by the following equation:

$$
J=\left\{\begin{array}{lll}
v_{\max } \rho & \text { if } & \rho<\rho_{c} \\
v_{\max } \rho_{c} \frac{(\rho-1)}{\left(\rho_{c}-1\right)} & \text { if } & \rho \geq \rho_{c}
\end{array}\right.
$$

Where $v_{\max }$ is the maximum velocity of the traffic and $\rho_{c}=\left(1+v_{\max }\right)^{-1}$ is the critical density. We consider the particular case of $v_{\max }=1$ where the maximum value of the flow is $J=0.5$, the corresponding critical density is $\rho_{c}=0.5$, which is the same value that corresponds to the transition of the metric $\Psi$ as illustrated in figure.3, thus the simulation of this particular case can be conducted using the metric $\Psi$. Given the fundamental equation of the flow and the average velocity $J=\langle v>\rho$ and given the property $\max (\Psi)=1$, we can define the velocity and the flow in terms of $\Psi$ by the following equations:

$$
\left\{\begin{array}{l}
J=\frac{\Psi}{2} \\
\langle v\rangle=\frac{\Psi}{2 \rho}
\end{array}\right.
$$

Further analysis of the function $\Psi$ can be employed to test its variation using a logistic map defined by the range of the sequence $0 \leq x_{n} \leq 1$ without using the binary transformation and comparatively to the Lyapunov exponents $\lambda$. A second possible extension consists of comparing the performance of the metric $\Psi$ with other predefined functions for randomness characterization of binary sequences using different parameters such as the length of the sequence and the density.

\section{Conclusion}

In this paper, we have studied the probabilistic characterization of one dimensional and binary cellular automaton model. Based on the density as the principal parameter, we 
have proposed a complex metric based on the average of the exponential factor to characterize the system. For two dimensional vector, this metric is equivalent to the XOR operator, and for large dimensional vector, the numerical results demonstrated that the metric separates two phases with linear rates and critical density of $\mathrm{p}=0.5$ which is the same result of the Shannon entropy function. In the second part, we have compared the variation of the metric using generated binary sequences obtained from a transformation of the logarithmic logistic map defined by the range of the growth parameter of $[0, e]$. The results illustrated the equivalence between the variations of the proposed metric and the information entropy where both functions are characterized by fluctuations in the chaotic region given by the range $[2.5, \mathrm{e}]$. As perspective, we have demonstrated an application of the proposed metric in particular case of the deterministic traffic flow.

\section{References}

[1] G. Vichniac, "Simulating physics with cellular automata", Physica D, vol. 10, (1984), pp. 96-115.

[2] R. M. Itami, "Simulating spatial dynamics: cellular automata theory", In Landscape and Urban Planning, ISSN 0169-2046, vol. 30, issues 1-2, (1994), pp. 27-47.

[3] R. White and G. Engelen, "Cellular dynamics and GIS: Modelling spatial complexity", Geographical Systems, (1994),pp. 1237-253.

[4] J. Kari, "Theory of cellular automata: A survey", In Theoretical Computer Science, ISSN 0304-3975, vol. 334, iss. 1-3, (2005), pp. 3-33.

[5] J. Esser and M. Schreckenberg, "Microscopic Simulation of Urban Traffic Based on Cellular Automata", International Journal of Modern Physics C, vol. 08, pp. 05, (1997), pp. 1025-1036.

[6] V. Belitsky and P.A. Ferrari, "Ballistic annihilation and deterministic surface growth", J Stat Physvol. 80, (1995), p. 517.

[7] P. Drabek, "Introduction to bifurcation theory", Topics in Mathematical Analysis (World Scientific), (2011), pp. 103-174.

[8] S. Wieczorek, B. Krauskopf, T. B. Simpson and D. Lenstra, Phys. Rep., vol. 416, no. 1, (2005).

[9] M. C. Gutzwiller, "Chaos in Classical and Quantum Mechanics", Springer-Verlag, New York, (1990).

[10] F. Xu and P. Tan, "Analysis of period-doubling bifurcation and chaos using physics-based SiC diode model", 2015 18th International Conference on Electrical Machines and Systems (ICEMS), Pattaya, (2015), pp. 612-615.

[11] R. H. Landau, M. J. Paez and C. C. Bordeianu, "Computational Physics, Problem Solving with Computers", 2nd Revised and Enlarged edn. (Wiley-VCH Verlag GmbH \& Co KGaA, Weinheim, (2007).

[12] Y. Totoki and T. Matsuo, "Decay/Growth Rate Estimation using Instantaneous Lyapunov Exponent", int. j. bifurcation chaos, vol. 22, no. 1250047, (2012).

[13] R. H. Landau, M. Jose Paez and C. C. Bordeianu, "A Survey of Computational Physics", Introductory Computational Science, Princeton University Press, (2008).

[14] Yo. Khmou, "A Numerical Study of New Logistic Map, Fluctuation and Noise Letters", (2018).

[15] S. Lbeck, M. Schreckenberg and K. D. Usadel, Phys. Rev. E, vol. 57, no. 1171, (1998).

[16] S Cheybani, J. Phys. A: Math. Gen., vol. 31, no. 9787, (1998). 